\title{
Blunted endocrine and cardiovascular reactivity in young healthy women reporting a history of childhood adversity
}

\author{
Annette Voellmin ${ }^{a, b}$, Katja Winzeler ${ }^{a, b}$, Evelin Hug ${ }^{a, b}$, \\ Frank H. Wilhelm ${ }^{c}$, Valérie Schaefer ${ }^{a}$, Jens Gaab ${ }^{b}$, \\ Roberto La Marca d, Jens C. Pruessner ${ }^{\mathrm{e}}$, Klaus Bader ${ }^{\mathrm{a}, *}$
}

\author{
a Psychiatric Clinics of the University of Basel, Center for Specific Psychotherapy, CBT Unit, Wilhelm \\ Klein-Strasse 27, 4012 Basel, Switzerland \\ b Department of Psychology, Division of Clinical Psychology and Psychotherapy, University of Basel, \\ Missionsstrasse 60/62, 4055 Basel, Switzerland \\ c University Salzburg, Department of Psychology, Division of Clinical Psychology, Psychotherapy, and Health \\ Psychology, Hellbrunnerstrasse 34, 5020 Salzburg, Austria \\ d Department of Psychology, Division of Clinical Psychology and Psychotherapy, University of Zurich, \\ Binzmuehlestrasse 14/26, 8050 Zurich, Switzerland \\ e Departments of Psychiatry, Neurology, and Neurosurgery, McGill University \& Douglas Hospital Research \\ Centre, Montreal, QC, Canada
}

\section{KEYWORDS}

Hypothalamuspituitary-adrenal axis; Sympathetic nervous system; Adverse childhood experiences;

Female; Stress reactivity; Resilience;

Trauma

\begin{abstract}
Summary
Background: Chronic or prolonged stress exposure in childhood can alter structural and functional brain development, leading to mental and physical illness and alterations of psychobiological stress systems in adulthood. Recently, attenuation in stress reactivity of the hypothalamic-pituitary-adrenal (HPA) axis and cardiovascular system have been related to the number of adverse childhood experiences (ACEs). We set out to investigate the association of ACE duration and age of ACE occurrence on stress reactivity.

Methods: 104 women in the age range $18-25$ years (mean $=21.7$ ) free of mental and physical illness underwent psychosocial stress testing with the Montreal Imaging Stress Task (MIST). Free saliva cortisol and heart rate were assessed repeatedly before and after the MIST.

Results: Number of ACEs was associated with attenuated cortisol and heart rate responses to stress in a dose-response relationship. Whereas overall duration of ACEs was significantly associated with an attenuated cortisol response, the specific age of first ACE occurrence did not contribute further to the dampened stress response.
\end{abstract}

\footnotetext{
* Corresponding author. Tel.: +4161325 51 19; fax: +4161325 7671 .
}

E-mail address: Klaus.Bader@upkbs.ch (K. Bader). 
Conclusions: ACEs are associated with blunted endocrine and cardiovascular stress reactivity in young and healthy women. Adverse life events in childhood, particularly if they occur repeatedly and chronically, show a strong association with alterations in stress reactivity in adulthood, potentially predisposing for later mental or physical disorders.

(c) 2014 Elsevier Ltd. All rights reserved.

\section{Introduction}

Adverse childhood experiences (ACEs), including physical, emotional and sexual abuse, affect a significant portion of the population and have been shown to be risk factors for the development and persistence of mental disorders such as depression, anxiety disorders, substance use disorders, or attention-deficit/hyperactivity disorder. Changes in stress sensitivity and functioning of the hypothalamic-pituitary-adrenal (HPA) axis have been suggested as causal factors (De Bellis, 2002; Tarullo and Gunnar, 2006; Heim et al., 2008). The HPA axis, together with the sympathetic nervous system (SNS), are key players in the formation of the stress response (Chrousos, 2009). Models of stress reactivity and health outcomes have therefore been a main focus in research towards understanding adversity and resilience processes. Different reactivity phenotype patterns have emerged in different studies, with a number of studies showing exaggerated HPA axis and SNS responses in the context of ACEs and psychopathology (Heim et al., 2000b; Heim and Nemeroff, 2001; Bremner et al., 2003; Rao et al., 2008).

In contrast to findings in clinical samples, a growing number of studies on healthy participants reported blunted endocrine (Carpenter et al., 2007, 2011; Elzinga et al., 2008; Lovallo et al., 2012) and cardiovascular (Lovallo et al., 2012) responses in association with ACEs. For example, Carpenter et al. (2007) reported blunted plasma cortisol responses to a psychosocial stress test in a healthy sample with a history of ACEs compared to participants without a history of childhood maltreatment. Also, a recent study by Lovallo et al. (2012) showed in a large sample of healthy participants diminished cortisol as well as heart rate responses with an increasing number of adverse life events, indicating an inverse dose-response relationship of ACEs and reactivity to a mental stress test.

To the best of our knowledge, the association of age of occurrence and duration of ACEs has not been investigated in healthy samples so far. There is evidence that these factors could have a differential impact on stress reactivity in adulthood (Tarullo and Gunnar, 2006; Schoedl et al., 2010; Tottenham and Sheridan, 2010). Since most studies used brief self-report questionnaires or life events checklists to assess ACEs (e.g. Carpenter et al., 2011; Lovallo et al., 2012), questions of duration of the events or the respective age when they happened widely remain unanswered. From a developmental perspective, age at traumatization is believed to be an important factor (Bosch et al., 2012). Brain components involved in stress response show large plasticity during pre- and postnatal periods and during early childhood, and some plasticity during later childhood and adolescence (Fumagalli et al., 2007; Andersen et al., 2008). Also, the duration of adverse experiences could be associated with psychobiological constructs. Particularly those environmental events that cause exceeding or prolonged stimulation of the stress system during these critical developmental periods could be linked to abnormal neurodevelopment and therefore be risk factors for lasting alterations in stress reactivity of the HPA axis and the SNS (Schoedl et al., 2010).

Therefore, the aim of the present study was to replicate the findings of attenuated endocrine and cardiovascular stress reactivity in association with a history of ACEs in a young, healthy, female adult sample. Furthermore, we aimed to investigate the association of duration as well as age of occurrence of adverse life events in childhood and adolescence with the stress reactivity in adulthood. To elucidate the association of age of occurrence and duration, in this study, the Early Trauma Inventory-Self Report (ETISR) served as a more detailed method in measuring ACEs (Bremner et al., 2007). The questionnaire retrospectively assesses a wide range of stress and trauma exposure before the age of 18 and considers age of occurrence as well as duration of the events.

In contrast to Lovallo et al. (2012) who employed standard public speaking and mental arithmetic stressors, and in contrast to the standardly used Trier social stress test (TSST, (Kirschbaum et al., 1993), we used the Montreal imaging stress task (MIST), that has been developed to be compatible with functional magnetic resonance brain imaging (Dedovic et al., 2005), but can be used in laboratory stress studies as well (La Marca et al., 2011). The MIST offers a promising alternative to conventional psychosocial stress tests. Advantages of the MIST are that participants sit still during the stress protocol and do not speak, therefore causing fewer artifacts measuring psychobiological parameters like heart rate or electrodermal activity. Furthermore, since the MIST is considered to evoke a moderate endocrine stress response, the observed findings represent HPA activity close to naturalistic settings (Smyth et al., 1998).

\section{Methods and materials}

\subsection{Participants}

The sample included 104 young and healthy females in the age of 18 to 25 years $(M=21.7 ; S D=1.5)$, recruited at three schools for health care professions and social work in Basel, Switzerland. The sample was part of an ongoing project, which included only female participants. The recruitment material referred to a 14-day sleep assessment describing the nature and prevalence of sleep and sleeping disorder and did not include explicit statements about childhood trauma or adverse childhood experiences.

Exclusion criteria were current physical or psychiatric illness, pregnancy, regular and heavy tobacco use $(>5$ 
cigarettes a day), the consumption of illegal drugs, and the use of medication that interferes with the central nervous or the adrenocorticoid system.

Furthermore, participants were requested to minimize physical exercise during the hour preceding the laboratory examination and not to take large meals, coffee, or cigarettes. For participants taking no oral contraceptives, the laboratory assessment was held in the luteal phase of the participant's menstrual cycle (Kirschbaum et al., 1999).

Participants received monetary compensation for their participation and provided written informed consent prior to participation. The ethical principles of the Declaration of Helsinki were followed and the study was accepted by the local Ethics Committee. All appointments took place in a laboratory of the Psychiatric Clinics of the University of Basel, Switzerland.

\subsection{Procedure}

After a preliminary screening assessment, participants reported to the laboratory for the stress examination, which took place between 3:30 pm and 6:00 pm to control for circadian variation and lasted for approximately $2.5 \mathrm{~h}$. Participants were told that the laboratory assessment would include a test on cognitive performance.

Upon arrival, participants were seated in a comfortable chair in front of a table with a computer screen and several magazines. After the heart rate sensors were attached, a ten minute resting period followed to customize participants with the laboratory. Then, a baseline measurement was conducted for five minutes. Immediately before the baseline measurement, participants provided the first saliva sample. Another saliva sample was collected immediately before participants engaged in the MIST. Following the stress exposure, a recovery period was conducted during which five additional salivary cortisol samples were collected together with self-report measures of the participants' emotional response to the stress task. At the end of the laboratory testing, participants were debriefed and signed a second written informed consent to approve the further use of their data.

\subsection{Stress induction}

The MIST (La Marca et al., 2011) was used to induce a psychosocial stress response. The MIST (Dedovic et al., 2005) is a standardized psychosocial stress test during which participants have to solve arithmetic tasks displayed on a computer screen under time pressure and social evaluation. The software adapts the difficulty of the tasks to the individual performance level of each participant, so that it is not possible to correctly answer more than $45-50 \%$ of the arithmetic tasks in the experimental condition. Participants had to complete three experimental runs, each lasting four minutes.

To induce a social evaluative threat, participants are told that their performance has to be close or equal to the average performance of a normative sample that is shown on the top of the computer screen, and that otherwise, their data cannot be used for the research purposes. Also, participants are informed that the study leader is watching their performance next door. Furthermore, after each of the first two runs, to further enhance social evaluative threat, the study leader informs participants that their performance is poor. In concrete terms, after the first experimental run, the experimenter calls the study leader to ask what to do in such an unusual situation. The participant then is told to repeat the task and to do better. Then, after the second unsuccessful performance, the slightly annoyed and demanding study leader enters the laboratory and interrogates the participant about her individual reasons for her poor performance. The study leader explains the high costs of the experiment in case of a possible exclusion if the participant does not achieve a better performance. Then, the last run of the MIST starts while the study leader stays in the room and watches the subject's performance standing right behind her.

These behaviors were standardized and practiced before study onset.

\subsection{Measures}

\subsubsection{Biological measures}

Saliva was collected at seven measurement points, whereof two took place before the stress test $(-10$ and $-1 \mathrm{~min})$ and five after the stress test $(+1,+10,+25,+40$ and +55 min $)$ using salivettes (Sarstedt, Sevelen, Switzerland). All saliva samples were first stored at $-22^{\circ} \mathrm{C}$, then thawed and centrifuged at $3000 \mathrm{rpm}$, before cortisol concentration in saliva was determined by enzyme immunoassay (ALPCO Diagnostics, Salem, USA). Because of unexpected high values, cortisol concentration was reanalyzed with a more established commercially available chemiluminescence immunoassay with high sensitivity (IBL International, Hamburg, Germany). The intra- and interassay coefficients for cortisol were below $8 \%$.

Heart rate was recorded using Vitaport 3 data acquisition system (TEMEC Instruments B.V., Netherlands). Electrocardiogram (ECG) recordings were taken using Lead-II electrode placement (RedDot ${ }^{T M}$, 2248-50, 3F Health Care, Germany) on the thorax with three disposable electrodes. A sampling rate of $1024 \mathrm{~Hz}$ was used for ECG recordings with a low pass filter of $512 \mathrm{~Hz}$ and a high pass filter of $0.5 \mathrm{~Hz}$. Anslab, a software for scientific analysis of physiological data, was used to analyze detected consecutive R-waves and calculate $\mathrm{R}-\mathrm{R}$ intervals, which were transformed to heart rate (Autonomic nervous system laboratory, Wilhelm \& Peyk, 2005). Heart rate was averaged for Baseline, Stress 1, Stress 2, Stress 3, and Recovery periods in reference to time markers manually set in accordance with the various sections of the experiment.

\subsubsection{Psychological measures}

A diagnostic screening including the German version of the "Structured Clinical Interview for DSM-IV/Axis I Disorders" (SCID-I) was conducted in order to detect and exclude participants suffering from a mental disorder (Wittchen et al., 1997). Lifetime history of mental disorders $(n=9)$ was assessed. Participants reported to have suffered from depression $(n=7)$, panic disorder $(n=1)$, and posttraumatic stress disorder comorbid with depression $(n=1)$ and were fully remitted at the time of the study. Relevant data including age, medication, drug consumption, age of menarche, 
Table 1 Participant characteristics and ACE scores of the study sample $(N=104)$.

\begin{tabular}{|c|c|c|c|}
\hline Variable & M & SD & Range/\% \\
\hline Age [yr] & 21.66 & 1.54 & $18-25$ \\
\hline Age of onset menarche ${ }^{\mathrm{a}}[\mathrm{yr}]$ & 12.95 & 1.28 & $10-16$ \\
\hline Oral contraceptive use, $n(\%)$ & & & $59(56.7)$ \\
\hline Body Mass Index $\left[\mathrm{kg} / \mathrm{m}^{2}\right]$ & 21.81 & 2.53 & $18.37-31.1$ \\
\hline Depressive symptoms (ADS-K) & 7.08 & 4.93 & $0-24$ \\
\hline ACE total sum score & 2.76 & 3.17 & $0-15$ \\
\hline General trauma & 1.43 & 1.63 & $0-7$ \\
\hline Physical abuse & .53 & .99 & $0-5$ \\
\hline Emotional abuse & .54 & 1.25 & $0-6$ \\
\hline Sexual abuse & .26 & .57 & $0-3$ \\
\hline $\mathrm{ACEs} \geq 1$ year & 1.27 & 2.40 & $0-12$ \\
\hline ACEs $<1$ year & 1.44 & 1.55 & $0-6$ \\
\hline ACEs before menarche & 1.96 & 2.99 & $0-15$ \\
\hline ACEs after menarche & .76 & 1.05 & $0-6$ \\
\hline Age of first ACE occurrence ${ }^{b}$ & 7.54 & 4.83 & $0-16$ \\
\hline
\end{tabular}

BMI, date of last menstruation, and intake of hormonal contraceptives were also assessed during the interview.

ACEs before the age of 18 years were assessed retrospectively using a German translation of the "Early Trauma Inventory-Self Report"' (ETI-SR) (Bremner et al., 2007), which includes general trauma (31 items), physical ( 9 items), emotional ( 7 items), and sexual abuse (15 items). The ETI-SR has been demonstrated to be a valid measure of early trauma, and has shown high internal consistency in all trauma domains (Cronbach $\alpha>0.7$ ) (Bremner et al., 2007).

Participants were asked a series of questions concerning potential trauma and stress exposure, which they answered with yes or no. Next, on positively answered items, age of occurrence, frequency of trauma or abuse, and emotional impact $(0=$ no negative impact, $1=$ slightly negative, 2 =moderately negative, $3=$ strongly negative) were assessed. In total, five different ACE scores were built. First, a sum score was computed from all events rated with an emotional impact of at least 1 (ACE total sum score). Furthermore, a sum score for ACEs lasting less than a year (ACEs< one year) and for ACEs lasting more than a year ( $A C E s>$ one year) was computed. Age of occurrence was assessed in two ways. In a first step, events which occurred before or after a participants' menarche were summed up to ACEs before and after menarche, respectively. Next, age of first ACE occurrence was abstracted for each subject, while the emotional impact of at least "slightly negative", was considered. ACE mean scores of the study sample are depicted in Table 1.

Because the ETI-SR does not provide cut-off scores for grouping, for illustration purposes, evenly distributed quartile groups (ACE total groups) were built via rank function of SPSS for the ACE total sum score. The grouping via rank function resulted in the following group distributions: group $1=0$ $\mathrm{ACE}$, group $2=1 \mathrm{ACE}$, group $3=2-3 \mathrm{ACEs}$, and group $4=4$ or more ACEs. Groups with regard to duration were then built according to the same group distribution as for the ACE total groups.
Depressive symptomatology was assessed via the German version of the Center for Epidemiological Studies Depression Scale (CES-D; German version: ADS-K; (Hautzinger and Bailer, 1993).

Visual analog scales (VAS) for mood, tension, and stress served as measures of subjective emotional response of participants during the psychosocial stress test. The scales ranged from "not stressed" (0) to "very stressed" (100), experiencing "no tension" (0) to "extreme tension" (1 00$)$, and "having a good mood" (0) to "having a bad mood" $(100)$, respectively.

\subsection{Data analysis}

Statistical analyses were performed using IBM SPSS Statistics, version 20 (SPSS Inc., Chicago, IL). Descriptive statistics were conducted for all variables. Skewed data were logarithmically transformed where appropriate.

First, repeated measures general linear model (GLM) was used to assess if the stress task led to a significant stress response for the dependent variables salivary cortisol, heart rate, as well as for the subjective emotional responses to the MIST. Next, GLMs for repeated measures served to determine the effects of ACEs on endocrine and cardiovascular responses. In these models, the ACE total sum score as well as the different scores for duration and age of occurrence were used as continuous variables to examine effects of time, ACE scores, and the interaction of time by ACE scores. In a second step, in order to visualize the results, the different ACE groups were then used as fixed factors for the GLMs, respectively.

To protect against violation of sphericity, Greenhouse-Geisser corrections were applied where appropriate. Effect sizes were determined by partial eta-square, reflecting small (.01), medium $(.06)$, or large (.14) effect sizes (Green et al., 2000).

To account for their potential confounding influence on cortisol concentration (Kirschbaum et al., 1999), BMI and 
use of oral contraceptives were included as covariates in all statistical models. In this sample, depressive symptoms were overall low and were neither related to cortisol, heart rate nor ACEs and therefore not controlled for in the analyses. Also, lifetime history of mental disorders $(n=9)$ was not related to the outcome measures and therefore not controlled for in the analyses. Emotional responses to the MIST (mood, tension, stress) were entered as covariates in post-hoc analyses, using the trapezoid formula for calculation (area under the curve with respect to ground, AUCg; Pruessner et al., 2003).

Technical difficulties with Vitaport 3 data acquisition system led to data loss in heart rate measurements (missing completely at random). Eventually, heart rate measures of 88 participants were available and went into the analyses. Cortisol data of four subjects had to be excluded because of unlikely high and fluctuating values, or because of acute illness, and therefore, cortisol measures of 100 participants went into the analyses.

\section{Results}

Demographic and trauma characteristics of the sample are displayed in Table 1. According to univariate analyses of variance, the ACE groups did not differ significantly in terms of demographic characteristics (e.g. age, age of onset of menarche, oral contraceptive use, and depressive symptoms). However, for the ACE total groups, BMI was significantly higher $[p=.02]$ in women reporting 4 or more ACEs $(M=23.38, S D=3.55)$ compared to women reporting no ACEs $(M=21.16, S D=2.07)$.

Results obtained by GLM repeated measure analyses indicated that the stress task induced a robust and significant increase in cortisol levels $[F(1.87,185.52)=27.16, p<.001$; $\left.\eta_{p}{ }^{2}=.22\right]$ and heart rate $[F(1.85,164.95)=216.86, p<.001$; $\left.\eta_{p}{ }^{2}=.71\right]$. Subjects experienced significant worsening of $\operatorname{mood}\left[F(3.53,360.36)=31.72, p<.001 ; \eta_{p}{ }^{2}=.24\right]$, as well as increases in tension $[F(3.78,385.39)=32.82, p<.001$; $\left.\eta_{p}{ }^{2}=.24\right]$, and stress $[F(3.90,398.15)=28.45, \quad p<.001$; $\left.\eta_{p}{ }^{2}=.22\right]$ (Fig. 1). Regarding their emotional reaction to the MIST, participants did not show differences in their baseline and peak levels in relation to the total number of ACEs, as indicated by univariate analyses of variances (data not shown).

Furthermore, the associations of the subjective emotional responses to the MIST with cortisol and heart rate responses were tested. Results revealed no significant correlations (all $p>1)$. Depression symptom scores and ACE total sum score were uncorrelated $(r=.11, p=.27)$.

\subsection{Association of ACE and cortisol responses to stress}

Repeated measures analysis of cortisol responses to stress showed a significant interaction of time $\times A C E$ total sum score $\left[F(2.33,221.60)=5.89, p<.001 ; \eta_{p}{ }^{2}=.06\right]$ as well as a significant main effect of $A C E$ total sum score $[F(1$, $\left.95)=7.52, p<.01 ; \eta_{p}{ }^{2}=.07\right]$. Results remained significant when the emotional responses to the stress task were entered additionally as covariates. Results are depicted in Fig. 2.

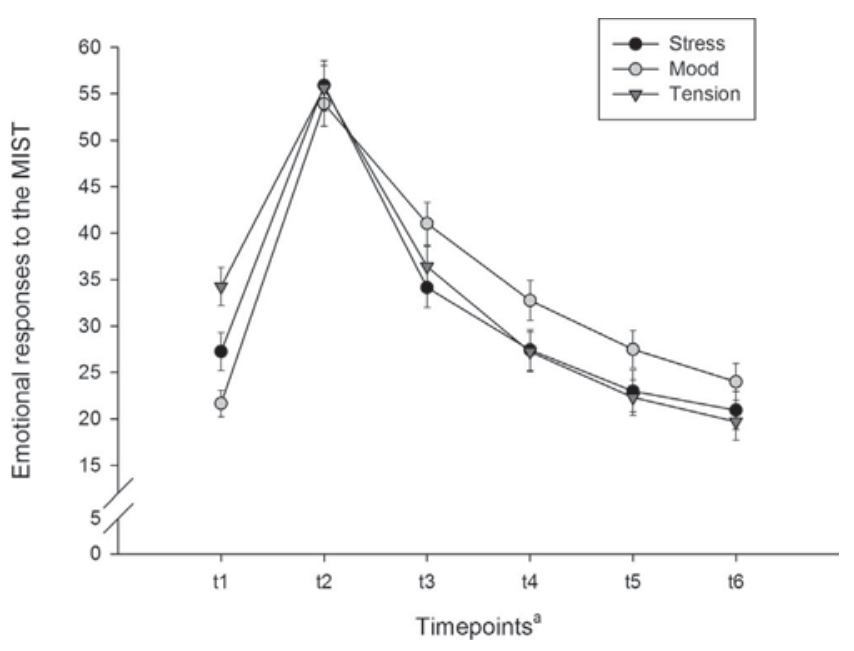

Fig. 1 Subjective emotional responses to the MIST. ( ${ }^{\mathrm{a}}$ Timepoints relative to the MIST: $t 1=-1 \mathrm{~min}, t 2=+1 \mathrm{~min}$, $t 3=+10 \mathrm{~min}, t 4=+25 \mathrm{~min}, t 5=+40 \mathrm{~min}, t 6=+55 \mathrm{~min}$. .

Next, it was tested whether the duration of ACEs was associated with the cortisol responses to the stress task. Repeated measures analysis of cortisol response to the stress task resulted in a significant main effect of duration of $A C E s>$ one year $\left[F(1,95)=10.10, p<.01 ; \eta_{p}{ }^{2}=.10\right]$ and a significant interaction of time and duration of $A C E s>$ one year $\left[F(2.33,221.16)=5.36, p<.01 ; \eta_{p}{ }^{2}=.05\right]$ (Fig. 3). Results remained significant when the emotional responses to the stress task were entered additionally as covariates. However, these effects were not observed for the association between ACEs that lasted shorter in duration (ACEs< one year) and cortisol responses to the stress task [main

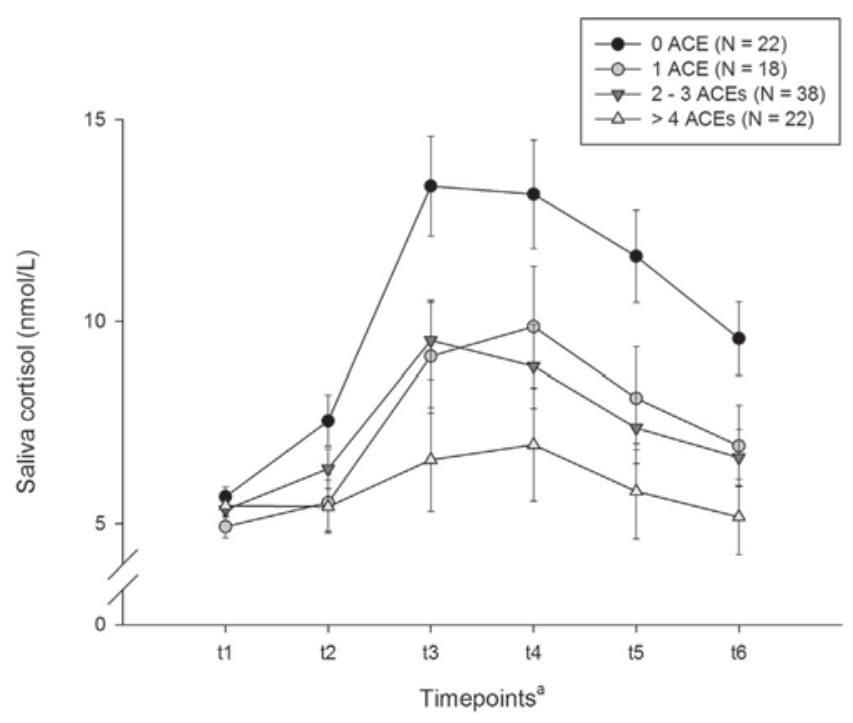

Fig. 2 Cortisol responses to the MIST are depicted for women who experienced $0,1,2-3$ or 4 or more ACEs. Values represent average cortisol \pm standard error of the mean for the ACE total groups controlled for oral contraceptive use and BMI. Higher ACE sum scores are associated with blunted cortisol responses. ( ${ }^{a}$ Timepoints relative to the MIST: $t 1=-1 \mathrm{~min}, t 2=+1 \mathrm{~min}$, $t 3=+10 \mathrm{~min}, t 4=+25 \mathrm{~min}, t 5=+40 \mathrm{~min}, t 6=+55 \mathrm{~min}$. . 


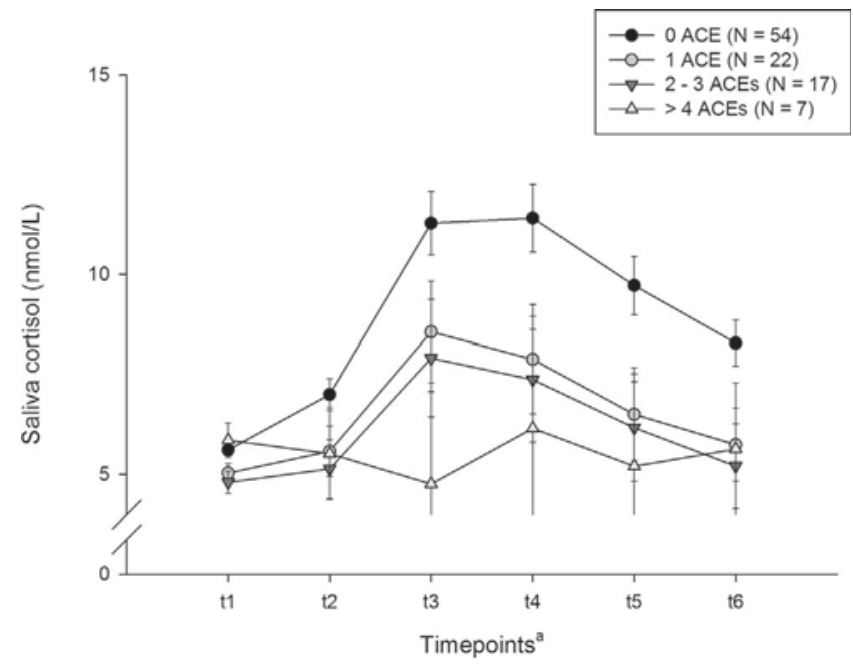

Fig. 3 Cortisol responses to the MIST are depicted for women who experienced $0,1,2-3$ or up to 4 or more ACEs that lasted more than a year. Values represent cortisol \pm standard error of the mean for the ACES > one year group controlled for oral contraceptive use and BMI. Higher ACE sum scores are associated with blunted cortisol responses. $\left({ }^{\mathrm{a}}\right.$ Timepoints relative to the MIST: $t 1=-1 \mathrm{~min}, t 2=+1 \mathrm{~min}, t 3=+10 \mathrm{~min}, t 4=+25 \mathrm{~min}$, $t 5=+40 \mathrm{~min}, t 6=+55 \mathrm{~min}$. ).

effect, $F(1,95)=.64, p=.43$; interaction effect, $F(2.24$, 213.21) $=1.05, p=.36]$.

For age of occurrence, a significant interaction effect $\left[F(2.33,220.99)=6.48, p<.01 ; \eta_{p}{ }^{2}=.06\right]$, and a significant main effect were observed for the sum of events which occurred before menarche $[F(1,95)=10.26, p<.01$; $\left.\eta_{p}{ }^{2}=.09\right]$. For events which occurred after menarche, these effects were not observed [main effect, $F(1,95)=.18$, $p=.67$; interaction effect, $F(2.24,212.34)=.63, p=.55]$. For the specific age of first ACE occurrence, no significant associations were observed [main effect, $F(1,78)=71, p=.40$; interaction effect, $F(2.24,174.75)=.56, p=.58]$.

\subsection{Association of ACE and heart rate reactivity to stress}

Repeated measures analysis of heart rate response to the stress task showed a significant main effect of ACE total sum score $\left[F(1,85)=7.13, p<.01 ; \eta_{p}{ }^{2}=.08\right]$ as well as a significant interaction effect of time $\times A C E$ total sum score $\left[F(1.98,168.32)=5.86, p<.01 ; \eta_{p}{ }^{2}=.07\right]$. Results remained significant, when the emotional responses to the stress task were entered as covariates (Fig. 4).

However, the further analyses with duration as well as age of occurrence of ACEs revealed no significant relationships with heart rate responses to the stress task (data not shown).

\section{Discussion}

We set out to assess the association of ACEs and psychobiological stress reactivity and its modulation by the number, duration and age of occurrence of ACEs in healthy young women. Our results are in line with previous reports of

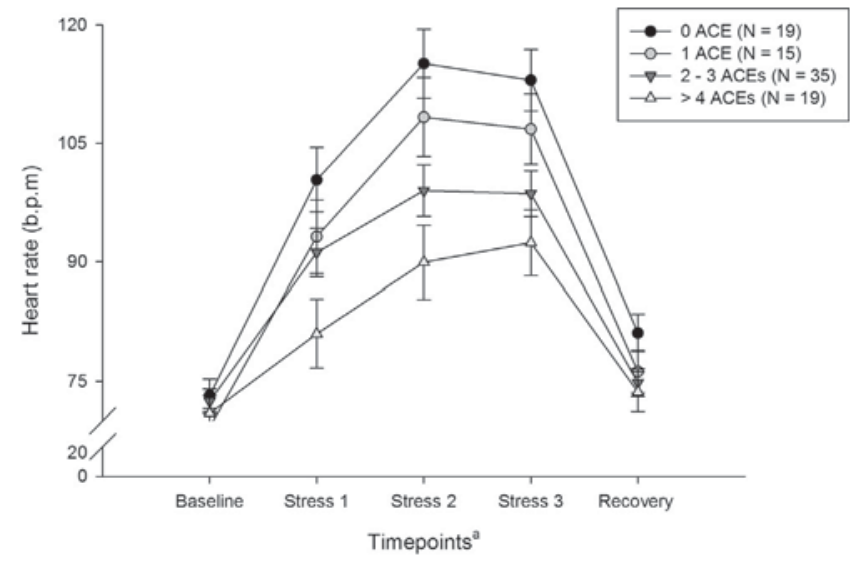

Fig. 4 Heart rate responses to the MIST are depicted for women who experienced $0,1,2-3$, or 4 or more ACEs. Values represent average heart rate (beats per minute) \pm standard error of the mean for the ACE total groups controlled for oral contraceptive use and BMI. Higher ACE sum scores are associated with attenuated heart rate reactivity. ( ${ }^{\text {a }}$ Timepoints relative to the MIST: Heart rate averaged for Baseline ( $-5 \mathrm{~min})$, Stress 1 (first run), Stress 2 (second run), Stress 3 (third run), and Recovery period (first 5 min after last run).).

attenuated endocrine (Carpenter et al., 2007, 2011; Elzinga et al., 2008; Lovallo et al., 2012) as well as cardiovascular (Lovallo et al., 2012) stress responses to a psychosocial stress test in healthy adults with a history of adverse childhood experiences. Furthermore, our results substantiate the importance of the mean number of ACEs on endocrine and cardiovascular response to psychosocial stress. Importantly, blunted cortisol and heart rate responses were independent of emotional responses, suggesting that the diminished endocrine and cardiovascular stress reactivity cannot be explained by a reduced emotional reaction to stress (which may be interpreted as flattened affect) after a history of childhood adversity.

To the best of our knowledge, the present study is the first to demonstrate that in healthy young women, especially long enduring, chronic ACEs show the strongest association with a blunted cortisol reactivity, adding valuable knowledge to the link between chronic childhood adversity and alterations of the HPA axis. Even though the sum of events which occurred before menarche showed an association with a blunted cortisol reactivity, whereas events after menarche did not, in this sample, the specific age of occurrence did not contribute to a further understanding of the association between timing of ACEs and endocrine and cardiovascular reactivity.

Per se, our results show a deviation from an expected endocrine and cardiovascular stress response in participants free of mental and physical illness in association with a history of ACEs. According to Obradovic (2012), taking together recent findings on stress reactivity in the context of early adversity, it is more accurate to state that exposure to early life stress may lead to dysregulated physiological phenotypes rather than to a particular pattern of hyper- or hyporesponsivity (Obradovic, 2012). The recently proposed adaptive calibration model (Del Giudice et al., 2011) offers an evolutionary-developmental theory of 
individual differences in physiological reactivity processes. The authors hypothesize that, at a very general level, a nonlinear relation between adverse life event exposure and stress response exists. However, in the context of high adversity, the model predicts an either vigilant profile, characterized by high biological stress responsiveness, or an unemotional, underresponsive profile, characterized by generally low HPA axis and SNS activity. Thus, these opposite phenotypes might be mediated by other factors and their interactions, e.g. the study sample, the type of maltreatment or the interaction of various environmental and genetic factors.

In terms of environmental factors, studies have demonstrated that the HPA axis in early human development is under strong social regulation (Tarullo and Gunnar, 2006). Therefore, several studies suggested parental caregiving as a moderator of the HPA reactivity (Gunnar et al., 1992; Nachmias et al., 1996; Tarullo and Gunnar, 2006). Thus, sensitive parenting appears to buffer cortisol responses in fearful situations, whereas being deprived of an evolutionarily expectable level of care (e.g. institutional rearing) has been associated with blunted cortisol production (Carlson and Earls, 1997; Gunnar et al., 2001). However, studies with institutionalized children were also able to show that improved caregiving environments had an effect on normalizing dampened HPA axis diurnal rhythms (Dozier et al., 2008; Cicchetti et al., 2011; Fisher et al., 2011).

The social buffering of the HPA axis is supported by findings in animal models. In their extensive summary of literature, Hostinar et al. (2014) report findings from animal studies, which suggest that neural mechanisms are responsible for behavioral and neuroendocrine changes due to social buffering (Hostinar et al., 2014). Findings from animal studies report changes in the development of the HPA axis and its function in social buffering. In early development, a stress hyporesponsive period (e.g. Witek-Janusek, 1988) during which the mother strongly controls the infants corticosterone levels has been reported, which can be disrupted by maternal deprivation and result in a hyperresponsive HPA pattern. In later stages of development, peers can also become a source of social buffering (Hennessy et al., 2009). For example, social input has been shown to dampen HPA axis reactivity in rat pups if the mother was present (e.g. Stanton and Levine, 1990; Shionoya et al., 2007), in maturing rodents when cohabitating companions are present (Terranova et al., 1999), or in squirrel monkeys in the presence of familiar and unfamiliar conspecifics (Vogt et al., 1981; Hennessy, 1984).

The findings in human and animal studies provide support for an adaptive response of the stress system to its environment, probably in order to enhance survival odds. It could be speculated that after an initial hypersecretion of cortisol due to chronic stressful environments, the HPA axis could counter-regulate its response and cortisol output might rebound to below normal. A plausible biological explanation could be an increased glucocorticoid negative feedback with a downregulation of CRF receptors, or a diminished release of cortisol by the adrenal glands (Heim et al., 2000a; Fries et al., 2005). This view is supported by our finding that only chronic events, not acute events, were associated with a blunted cortisol response.
Recent studies link genetic and epigenetic alterations to stress reactivity in association with ACEs as well. A history of ACEs has been associated with an epigenetic regulation of the glucocorticoid receptor in the hippocampus (McGowan et al., 2009). Moreover, a recent study on healthy adults who experienced the loss of a parent during childhood, maltreatment, or low parental care showed epigenetic alterations of a region of the human glucocorticoid receptor gene, which in turn was associated with a blunted cortisol reactivity after a neuroendocrine challenge test in these participants (Tyrka et al., 2012). Another study linked prenatal maternal depression to increased methylation of the glucocorticoid receptor gene, and showed exaggerated salivary cortisol output to stress at 3 months of age (Oberlander et al., 2008). Further studies are needed to investigate these changes in central regulation of the glucocorticoid receptor in brain regions involved in stress responses in association to ACEs.

Our results raise the challenging question of whether the observed alterations in stress responsivity can be interpreted as a potential risk factor for or as a sign of resilience to the development of later mental and physical disorders. Even if initially adaptive, blunted cortisol reactivity could compromise future and necessary psychobiological stress reactivity. For example, low cardiovascular and/or endocrine reactivity to acute psychological stress has been associated with depression, fibromyalgia, obesity, burn out, substance use disorders, and chronic pain syndromes (Griep et al., 1998; Heim et al., 1998; Pruessner et al., 1999; Lovallo et al., 2000; Gold and Chrousos, 2002; Gur et al., 2004; Phillips et al., 2011; Jones et al., 2012).

Considering that participants in the present study were recruited from a school of higher education and were free of psychopathology in adulthood, they may have been selected in a way that the blunted stress reactivity pattern may stand for resilience to the development of mental illness in the aftermath of childhood adverse experiences. Longitudinal, population-based research is needed to investigate if participants without present psychopathology, who had shown blunted stress responses after stress induction, are at higher risk to develop psychiatric disorders later in life or remain healthy.

The present study revealed diminished heart rate responses in association with the total number of ACEs, but not with the subgroups regarding duration and age of occurrence. Recently, Bauer \& Boyce have suggested the examination of the HPA axis and the SNS simultaneously for a better understanding of their coordination (additive or interactive; or opposing or complementary) (Bauer et al., 2002). Only few studies have examined the exact nature of their coordination in adult samples so far (Ali and Pruessner, 2012; Lovallo et al., 2012; Andrews and Pruessner, 2013), and results are mixed. Methodological differences between the reported studies could account for the different findings. More empirical research is needed to investigate the exact nature of SNS alterations after ACEs, as well as the coordination and interactions between the two stress systems.

Our results revealed no associations of the specific age of occurrence, which contrasts to findings of other studies. Bosch et al. (2012) reported that especially ACEs in preand postnatal developmental stages were associated with heightened cortisol reactivity (Bosch et al., 2012). However, their sample included 16-year old adolescents, and also 
included concurrent psychopathology. In contrast to the present study, which used age of first ACE occurrence, and the distinction pre- and postmenarche, the authors used more distinct age groups to examine the association of age and cortisol reactivity. A limitation of our approach, by using the specific age of occurrence, is that type of trauma is neglected. Type of trauma is an important factor, which could also explain different HPA axis phenotypes. Future studies are needed to further analyze timing of ACEs, and methodologically, this might be assessed best in a sample of participants with one specific severe trauma/life event. Furthermore, a limitation of the present study is that no data on pre- and postnatal stress exposure was included. Prenatal maternal stress (Entringer et al., 2009) and postnatal adversity (Bosch et al., 2012) have been associated with increased HPA responses to stress, which could result in a sensitization to stressors in long-term.

Another limitation of the present study is that only peripheral readouts of stress hormone activation were measured. The HPA axis and the cardiovascular system are complex and multilayer systems and therefore we are not able to identify the exact mechanisms or location of the observed dysregulations. Furthermore, that only women were recruited and tested has to be mentioned as another potentially limitating factor. Especially as some of the recent models on stress reactivity changes after chronic or traumatic stress make mention of sex differences, it would have been informative to assess the stress response in men as well (Bangasser, 2013). Thus, due to the characteristics of the study sample, the presented findings can only be generalized to young women free of mental and physical illness. Also, participants were attendees of schools for health care professions and social work, which could have led to a selection bias as outlined above. Also, even though Hardt and Rutter (2004) concluded in their review of studies from 1980 to 2001 that the validity of retrospective recall of sexual/physical abuse, physical/emotional neglect or family discord is sufficiently valid (Hardt and Rutter, 2004). Still, the retrospectively assessed adversities could have been underestimated and/or biased, especially reported events in toddler and pre-verbal ages.

Because of methodological reasons, the specification between ACEs shorter/longer than one year cannot be assumed to be completely independent from each other. Since some participants reported both, acute and chronic ACEs, with our sample size, it was not possible to have fully distinct and statistically orthogonal groups.

Despite these limitations, the present study strengthens the assumption that adverse childhood experiences give rise to a blunted stress reactivity of the HPA axis and the SNS in young healthy women. In this study population, number and duration of adverse events in childhood showed the strongest association with an attenuated stress response in adulthood. These findings suggest that the reactivity of the human stress system is indeed shaped by the experience of extrinsic chronic stressors in childhood and adolescence.

\section{Contributors}

Klaus Bader (PI) and Frank $\mathrm{H}$. Wilhelm designed the study and wrote the protocol.
Jens C. Pruessner developed the Montreal Imaging Stress Task and together with Roberto La Marca trained us in how to use it and supervised us in administrating the stress protocol outside the MRT.

Evelin Hug wrote the first draft of the paper, Annette Voellmin wrote the final draft of the manuscript and undertook the statistical analysis. Annette Voellmin managed the recruitment of the participants, together with Katja Winzeler, who was involved in the literature research and the proof reading of the manuscript.

Valérie Schaefer helped in the recruitment of the participants and was also part of the study design team.

Jens Gaab assisted Annette Voellmin in the manuscript preparation and in the statistical analyses.

\section{Role of the funding source}

This study was supported by Swiss National Science Foundation (SNSF) grant \#100014_126635/1 (KB, FHW).

\section{Conflict of interest statement}

The authors have no biomedical financial interests or potential conflicts of interest to report.

\section{Acknowledgements}

We thank the participants of this study, as well as the graduate students from the University of Basel for their assistance. We also thank Dr. Andrea Meyer for his statistical assistance and Dr. Christian Cajochen for his contributions to the study design.

\section{References}

Ali, N., Pruessner, J.C., 2012. The salivary alpha amylase over cortisol ratio as a marker to assess dysregulation of the stress system. Physiol. Behav. 106, 65-72.

Andersen, S.L., Tomada, A., Vincow, E.S., Valente, E., Polcari, A., Teicher, M.H., 2008. Preliminary evidence for sensitive periods in the effect of childhood sexual abuse on regional brain development. J. Neuropsychiatry Clin. Neurosci. 20, 292-301.

Andrews, J., Pruessner, J., 2013. The combined propanol/TSST paradigm - a new method for psychoneuroendocrinology. PLoS One 8 (2), e57567.

Bangasser, D.A., 2013. Sex differences in stress-related receptors: "micro" differences with "macro" implications for mood and anxiety disorders. Biol. Sex Differ. 4, 2.

Bauer, A.M., Quas, J.A., Boyce, W.T., 2002. Associations between physiological reactivity and children's behavior: advantages of a multisystem approach. J. Dev. Behav. Pediatr. 23, 102-113.

Bosch, N.M., Riese, H., Reijneveld, S.A., Bakker, M.P., Verhulst, F.C., Ormel, J., Oldehinkel, A.J., 2012. Timing matters: long term effects of adversities from prenatal period up to adolescence on adolescents' cortisol stress response. The TRAILS study. Psychoneuroendocrinology 37, 1439-1447.

Bremner, J.D., Bolus, R., Mayer, E.A., 2007. Psychometric properties of the early trauma inventory-self report. J. Nerv. Ment. Dis. 195, $211-218$.

Bremner, J.D., Vythilingam, M., Vermetten, E., Adil, J., Khan, S., Nazeer, A., Afzal, N., McGlashan, T., Elzinga, B., Anderson, G.M., Heninger, G., Southwick, S.M., Charney, D.S., 2003. 
Cortisol response to a cognitive stress challenge in posttraumatic stress disorder (PTSD) related to childhood abuse. Psychoneuroendocrinology 28, 733-750.

Carlson, M., Earls, F., 1997. Psychological and neuroendocrinological sequelae of early social deprivation in institutionalized children in Romania. Ann. N.Y. Acad. Sci. 807, 419-428.

Carpenter, L.L., Carvalho, J.P., Tyrka, A.R., Wier, L.M., Mello, A.F., Mello, M.F., Anderson, G.M., Wilkinson, C.W., Price, L.H., 2007. Decreased adrenocorticotropic hormone and cortisol responses to stress in healthy adults reporting significant childhood maltreatment. Biol. Psychiatry 62, 1080-1087.

Carpenter, L.L., Shattuck, T.T., Tyrka, A.R., Geracioti, T.D., Price, L.H., 2011. Effect of childhood physical abuse on cortisol stress response. Psychopharmacology (Berl) 214, 367-375.

Chrousos, G.P., 2009. Stress and disorders of the stress system. Nat. Rev. Endocrinol. 5, 374-381.

Cicchetti, D., Rogosch, F.A., Toth, S.L., Sturge-Apple, M.L., 2011. Normalizing the development of cortisol regulation in maltreated infants through preventive interventions. Dev. Psychopathol. 23, 789-800.

De Bellis, M.D., 2002. Developmental traumatology: a contributory mechanism for alcohol and substance use disorders. Psychoneuroendocrinology 27, 155-170.

Dedovic, K., Renwick, R., Mahani, N.K., Engert, V., Lupien, S.J., Pruessner, J.C., 2005. The Montreal imaging stress task: using functional imaging to investigate the effects of perceiving and processing psychosocial stress in the human brain. J. Psychiatry Neurosci. 30, 319-325.

Del Giudice, M., Ellis, B.J., Shirtcliff, E.A., 2011. The adaptive calibration model of stress responsivity. Neurosci. Biobehav. Rev. 35, 1562-1592.

Dozier, M., Peloso, E., Lewis, E., Laurenceau, J.P., Levine, S., 2008. Effects of an attachment-based intervention on the cortisol production of infants and toddlers in foster care. Dev. Psychopathol. 20, 845-859.

Elzinga, B.M., Roelofs, K., Tollenaar, M.S., Bakvis, P., van Pelt, J., Spinhoven, P., 2008. Diminished cortisol responses to psychosocial stress associated with lifetime adverse events a study among healthy young subjects. Psychoneuroendocrinology 33, 227-237.

Entringer, S., Kumsta, R., Hellhammer, D.H., Wadhwa, P.D., Wust, S., 2009. Prenatal exposure to maternal psychosocial stress and HPA axis regulation in young adults. Horm. Behav. 55, 292-298.

Fisher, P.A., Van Ryzin, M.J., Gunnar, M.R., 2011. Mitigating HPA axis dysregulation associated with placement changes in foster care. Psychoneuroendocrinology 36, 531-539.

Fries, E., Hesse, J., Hellhammer, J., Hellhammer, D.H., 2005. A new view on hypocortisolism. Psychoneuroendocrinology 30, 1010-1016.

Fumagalli, F., Molteni, R., Racagni, G., Riva, M.A., 2007. Stress during development: impact on neuroplasticity and relevance to psychopathology. Prog. Neurobiol. 81, 197-217.

Gold, P.W., Chrousos, G.P., 2002. Organization of the stress system and its dysregulation in melancholic and atypical depression: high vs low CRH/NE states. Mol. Psychiatry 7, 254-275.

Green, S.B., Salkind, N.J., Akey, T.M., 2000. Using SPSS for Windows, second ed. Prentice Hall, Englewood Cliffs, NJ.

Griep, E.N., Boersma, J.W., Lentjes, E.G., Prins, A.P., van der Korst, J.K., de Kloet, E.R., 1998. Function of the hypothalamic-pituitary-adrenal axis in patients with fibromyalgia and low back pain. J. Rheumatol. 25, 1374-1381.

Gunnar, M.R., Larson, M.C., Hertsgaard, L., Harris, M.L., Brodersen, L., 1992. The stressfulness of separation among nine-month-old infants: effects of social context variables and infant temperament. Child Dev. 63, 290-303.
Gunnar, M.R., Morison, S.J., Chisholm, K., Schuder, M., 2001. Salivary cortisol levels in children adopted from Romanian orphanages. Dev. Psychopathol. 13, 611-628.

Gur, A., Cevik, R., Nas, K., Colpan, L., Sarac, S., 2004. Cortisol and hypothalamic-pituitary-gonadal axis hormones in follicularphase women with fibromyalgia and chronic fatigue syndrome and effect of depressive symptoms on these hormones. Arthritis Res. Ther. 6, R232-R238.

Hardt, J., Rutter, M., 2004. Validity of adult retrospective reports of adverse childhood experiences: review of the evidence. J. Child Psychol. Psychiatry 45, 260-273.

Hautzinger, M., Bailer, M., 1993. Allgemeine Depressions-Skala (ADS): Manual. Beltz Test, Weinheim.

Heim, C., Ehlert, U., Hanker, J.P., Hellhammer, D.H., 1998. Abuserelated posttraumatic stress disorder and alterations of the hypothalamic-pituitary-adrenal axis in women with chronic pelvic pain. Psychosom. Med. 60, 309-318.

Heim, C., Ehlert, U., Hellhammer, D.H., 2000a. The potential role of hypocortisolism in the pathophysiology of stress-related bodily disorders. Psychoneuroendocrinology 25, 1-35.

Heim, C., Nemeroff, C.B., 2001. The role of childhood trauma in the neurobiology of mood and anxiety disorders: preclinical and clinical studies. Biol. Psychiatry 49, 1023-1039.

Heim, C., Newport, D.J., Heit, S., Graham, Y.P., Wilcox, M., Bonsall, R., Miller, A.H., Nemeroff, C.B., 2000b. Pituitary-adrenal and autonomic responses to stress in women after sexual and physical abuse in childhood. JAMA 284, 592-597.

Heim, C., Newport, D.J., Mletzko, T., Miller, A.H., Nemeroff, C.B., 2008. The link between childhood trauma and depression: insights from HPA axis studies in humans. Psychoneuroendocrinology 33, 693-710.

Hennessy, M.B., 1984. Presence of companion moderates arousal of monkeys with restricted social experience. Physiol. Behav. 33, 693-698.

Hennessy, M.B., Kaiser, S., Sachser, N., 2009. Social buffering of the stress response: diversity, mechanisms, and functions. Front. Neuroendocrinol. 30, 470-482.

Hostinar, C.E., Sullivan, R.M., Gunnar, M.R., 2014. Psychobiological mechanisms underlying the social buffering of the hypothalamic-pituitary-adrenocortical axis: a review of animal models and human studies across development. Psychol. Bull. 140, 256-282.

Jones, A., McMillan, M.R., Jones, R.W., Kowalik, G.T., Steeden, J.A., Deanfield, J.E., Pruessner, J.C., Taylor, A.M., Muthurangu, V., 2012. Adiposity is associated with blunted cardiovascular, neuroendocrine and cognitive responses to acute mental stress. PLoS One 7, e39143.

Kirschbaum, C., Kudielka, B.M., Gaab, J., Schommer, N.C., Hellhammer, D.H., 1999. Impact of gender, menstrual cycle phase, and oral contraceptives on the activity of the hypothalamus-pituitary-adrenal axis. Psychosom. Med. 61, 154-162.

Kirschbaum, C., Pirke, K.M., Hellhammer, D.H., 1993. The 'Trier Social Stress Test'-a tool for investigating psychobiological stress responses in a laboratory setting. Neuropsychobiology 28 , 76-81.

La Marca, R., Waldvogel, P., Thorn, H., Tripod, M., Wirtz, P.H., Pruessner, J.C., Ehlert, U., 2011. Association between cold face test-induced vagal inhibition and cortisol response to acute stress. Psychophysiology 48, 420-429.

Lovallo, W.R., Dickensheets, S.L., Myers, D.A., Thomas, T.L., Nixon, S.J., 2000. Blunted stress cortisol response in abstinent alcoholic and polysubstance-abusing men. Alcohol. Clin. Exp. Res. 24, 651-658.

Lovallo, W.R., Farag, N.H., Sorocco, K.H., Cohoon, A.J., Vincent, A.S., 2012. Lifetime adversity leads to blunted stress axis reactivity: studies from the Oklahoma Family Health Patterns Project. Biol. Psychiatry 71, 344-349. 
McGowan, P.O., Sasaki, A., D’Alessio, A.C., Dymov, S., Labonte, B., Szyf, M., Turecki, G., Meaney, M.J., 2009. Epigenetic regulation of the glucocorticoid receptor in human brain associates with childhood abuse. Nat. Neurosci. 12, 342-348.

Nachmias, M., Gunnar, M., Mangelsdorf, S., Parritz, R.H., Buss, K., 1996. Behavioral inhibition and stress reactivity: the moderating role of attachment security. Child Dev. 67, 508-522.

Oberlander, T.F., Weinberg, J., Papsdorf, M., Grunau, R., Misri, S., Devlin, A.M., 2008. Prenatal exposure to maternal depression, neonatal methylation of human glucocorticoid receptor gene (NR3C1) and infant cortisol stress responses. Epigenetics 3, 97-106.

Obradovic, J., 2012. How can the study of physiological reactivity contribute to our understanding of adversity and resilience processes in development? Dev. Psychopathol. 24, 371-387.

Phillips, A.C., Hunt, K., Der, G., Carroll, D., 2011. Blunted cardiac reactions to acute psychological stress predict symptoms of depression five years later: evidence from a large community study. Psychophysiology 48, 142-148.

Pruessner, J.C., Hellhammer, D.H., Kirschbaum, C., 1999. Burnout, perceived stress, and cortisol responses to awakening. Psychosom. Med. 61, 197-204.

Pruessner, J.C., Kirschbaum, C., Meinlschmid, G., Hellhammer, D.H., 2003. Two formulas for computation of the area under the curve represent measures of total hormone concentration versus time-dependent change. Psychoneuroendocrinology 28, 916-931.

Rao, U., Hammen, C., Ortiz, L.R., Chen, L.A., Poland, R.E., 2008. Effects of early and recent adverse experiences on adrenal response to psychosocial stress in depressed adolescents. Biol. Psychiatry 64, 521-526.

Schoedl, A.F., Costa, M.C., Mari, J.J., Mello, M.F., Tyrka, A.R., Carpenter, L.L., Price, L.H., 2010. The clinical correlates of reported childhood sexual abuse: an association between age at trauma onset and severity of depression and PTSD in adults. J. Child Sex Abuse 19, 156-170.

Shionoya, K., Moriceau, S., Bradstock, P., Sullivan, R.M., 2007. Maternal attenuation of hypothalamic paraventricular nucleus norepinephrine switches avoidance learning to preference learning in preweanling rat pups. Horm. Behav. 52, 391-400.

Smyth, J., Ockenfels, M.C., Porter, L., Kirschbaum, C., Hellhammer, D.H., Stone, A.A., 1998. Stressors and mood measured on a momentary basis are associated with salivary cortisol secretion. Psychoneuroendocrinology 23, 353-370.

Stanton, M.E., Levine, S., 1990. Inhibition of infant glucocorticoid stress response: specific role of maternal cues. Dev. Psychobiol. 23, 411-426.

Tarullo, A.R., Gunnar, M.R., 2006. Child maltreatment and the developing HPA axis. Horm. Behav. 50, 632-639.

Terranova, M.L., Cirulli, F., Laviola, G., 1999. Behavioral and hormonal effects of partner familiarity in periadolescent rat pairs upon novelty exposure. Psychoneuroendocrinology 24, 639-656.

Tottenham, N., Sheridan, M.A., 2010. A review of adversity, the amygdala and the hippocampus: a consideration of developmental timing. Front. Hum. Neurosci. 3, 68.

Tyrka, A.R., Price, L.H., Marsit, C., Walters, O.C., Carpenter, L.L., 2012. Childhood adversity and epigenetic modulation of the leukocyte glucocorticoid receptor: preliminary findings in healthy adults. PLoS One 7, e30148.

Vogt, J.L., Coe, C.L., Levine, S., 1981. Behavioral and adrenocorticoid responsiveness of squirrel monkeys to a live snake: is flight necessarily stressful? Behav. Neural Biol. 32, 391-405.

Witek-Janusek, L., 1988. Pituitary-adrenal response to bacterial endotoxin in developing rats. Am. J. Physiol. 255, E525-E530.

Wittchen, H.-U., Wunderlich, U., Gruschwitz, S., Zaudig, M., 1997. SKID-I: Strukturiertes Klinisches Interview für DSM-IV-Achse I: Psychische Störungen. Hogrefe, Göttingen. 